\title{
Volumetric Differences in Mapped Hippocampal Regions Correlate with Increase of High Alpha Rhythm in Alzheimer's Disease
}

\author{
D. V. Moretti, ${ }^{1}$ A. Prestia, ${ }^{1}$ C. Fracassi, ${ }^{1}$ C. Geroldi, ${ }^{1}$ G. Binetti, ${ }^{1}$ P. M. Rossini, ${ }^{2}$ \\ O. Zanetti, ${ }^{1}$ and G. B. Frisoni ${ }^{1}$
}

${ }^{1}$ IRCCS S. Giovanni di Dio Fatebenefratelli, 4, Pilastroni Road, 25125 Brescia, Italy

${ }^{2}$ Catholic University of the Holy Heart, largo Agostino Gemelli 1, 00168 Rome, Italy

Correspondence should be addressed to D. V. Moretti, davide.moretti@afar.it

Received 27 September 2010; Revised 12 April 2011; Accepted 13 April 2011

Academic Editor: Katsuya Urakami

Copyright ( 2011 D. V. Moretti et al. This is an open access article distributed under the Creative Commons Attribution License, which permits unrestricted use, distribution, and reproduction in any medium, provided the original work is properly cited.

Objective. The increase of high alpha relative to low alpha power has been recently demonstrated as a reliable EEG marker of hippocampal atrophy conversion of patients with mild cognitive impairment (MCI) in Alzheimer's disease (AD). In the present study we test the reliability of this EEG index in subjects with AD. Methods. Correlation between EEG markers and volumetric differences in mapped hippocampal regions was estimated in AD patients. Results. Results show that the increase of alpha3/alpha2 power ratio is correlated with atrophy of mapped hippocampal regions in Alzheimer's disease. Conclusions. The findings confirm the possible diagnostic role of EEG markers.

\section{Introduction}

Recent studies have demonstrated that hippocampus is not a unitary structure [1]. The hippocampus, including strictly speaking subfields CA1-CA4, and the hippocampal formation, including also dentate gyrus, fimbria, subiculum, and parasubiculum, is a highly sophisticated structure. Stimuli coming from the entorhinal cortex are processed by the dentate gyrus, subfields CA4 and CA3, before being projected outside the medial temporal lobe via CA1 or subicular efferent projections. Moreover, in addition to the unsurprising right-left specialization for verbal and visuospatial material [2], some degree of anterior-to-posterior specialization has been shown by fMRI studies [3].

As a consequence, it is conceivable that local structural changes take place in the hippocampus of patients with Alzheimer's disease (AD) and that different hippocampal subregions are affected in $\mathrm{AD}$. Local changes in hippocampal subregions could be detected through a radial atrophy mapping method able to assess group, based on high resolution MRI at 3 Tesla differences ([1] Frisoni et al. 2008).

A large body of literature has previously demonstrated that in subjects with cognitive decline are present specific modifications of brain rhythms detected by electroencefalography (EEG; [4-11]). In particular, an increase of high alpha as compared to low alpha band has been demonstrated to correlate specifically with hippocampal atrophy ([10] Moretti et al. 2009a) in subjects with mild cognitive impairment (MCI). Moreover, a recent study has shown that the increase of alpha3/alpha2 power ratio is a reliable index to predict MCI patients who will convert in AD [12]. So, the reliability of this EEG marker needs further confirmation in patients with $\mathrm{AD}$, and, if it is possible, with a greater topographic specificity.

In this study we tested the hypothesis that the increase of alpha3/alpha2 ratio is related with volumetric differences in mapped hippocampal regions in $\mathrm{AD}$ patients. Up to date, the correlation between mapped hippocampal regions and EEG activity has never been carried out in Alzheimer's disease.

\section{Materials and Methods}

2.1. Subjects. For the present study, 13 subjects with Alzheimer's disease $(\mathrm{AD})$ were recruited from the memory Clinic of the Scientific Institute for Research and Care 
(IRCCS) of Alzheimer's and psychiatric diseases "Fatebenefratelli" in Brescia, Italy. All experimental protocols had been approved by the local Ethics Committee. Informed consent was obtained from all participants or their caregivers, according to the Code of Ethics of the World Medical Association (Declaration of Helsinki). The diagnosis of $\mathrm{AD}$ was made according to NINCDS-ADRDA criteria [13] and the Diagnostic and Statistical Manual of Mental Disorders IV [14].

2.1.1. EEG Recordings. All recordings were obtained in the morning with subjects resting comfortably. In order to keep the level of vigilance constant, an operator controlled the subject and the EEG traces on-line, alerting the subject any time there were signs of behavioural and/or EEG drowsiness.

The EEG activity was recorded continuously from 19 sites by using electrodes set in an elastic cap (ElectroCap International, Inc.) and positioned according to the 10-20 International system (Fp1, Fp2, F7, F3, Fz, F4, F8, T3, C3, Cz, C4, T4, T5, P3, Pz, P4, T6, O1, O2). The ground electrode was placed in front of Fz. The left and right mastoids served as linked-mastoid reference for all electrodes. The recordings were used off-line to rereference the scalp recordings to the common average. Data were recorded with a bandpass filter of $0.3-70 \mathrm{~Hz}$ and digitized at a sampling rate of $250 \mathrm{~Hz}$ (BrainAmp, BrainProducts, Germany). Electrodes-skin impedance was set below $5 \mathrm{k} \Omega$. Horizontal and vertical eye movements were detected by recording the electrooculogram (EOG). The recording lasted 5 minutes, with subjects with closed eyes. Longer recordings would have reduced the variability of the data, but they would also have increased the possibility of slowing of EEG oscillations due to reduced vigilance and arousal. EEG data were then analyzed and fragmented off-line in consecutive epochs of 2 seconds, with a frequency resolution of $0.5 \mathrm{~Hz}$. The average number of epochs analyzed was 140 ranging from 130 to 150 . The EEG epochs with ocular, muscular, and other types of artifact were preliminary identified by a computerized automatic procedure [15]. A threshold of $\pm 50 \mu \mathrm{V}$ was set to detect muscular artifact. Two expert electroencephalographists manually double-checked and confirmed the automatic selections.

The EEG epochs with ocular, muscular, and other types of artifacts were discarded. The estimation of frequency band range was based on the individually detected spectral peak of the main EEG frequencies. The frequency peak, within a well defined band of interest, is the expression of the synchronization of a functional neuronal population [16, 17]. In this view, the frequency range so obtained could be reasonably less affected by slow or fast artefacts such as, respectively, ocular or muscular activity [15] as well as manifestations of miniature saccades [18].

2.1.2. Analysis of Individual Frequency Bands. A digital FFTbased power spectrum analysis (Welch technique, Hanning windowing function, no phase shift) computed-ranging from 2 to $45 \mathrm{~Hz}$ - the power density of EEG rhythms with a $0.5 \mathrm{~Hz}$ frequency resolution. Two anchor frequencies were selected according to literature guidelines [19], that is, the theta/alpha transition frequency (TF) and the individual alpha frequency (IAF) peak. The TF marks the transition frequency between the theta and alpha bands and represents an estimate of the frequency at which the theta and alpha spectra intersect. TF was computed as the minimum power in the alpha frequency range, since our EEG recordings were performed at rest. The IAF represents the frequency with the maximum power peak within the extended alpha range (5$14 \mathrm{~Hz}$ ). Based on TF and IAF, we estimated the frequency band range for each subject, as follows: delta from TF-4 to TF-2, theta from TF-2 to TF, low alpha band (alpha1 and alpha2) from TF to IAF, and high alpha band (or alpha3) from IAF to $I A F+2$. The alpha1 and alpha 2 bands were computed for each subject as follows: alphal from TF to the middle point of the TF-IAF range, and alpha2 from such middle point to the IAF peak $[6-9,20]$. Moreover, individual beta and gamma frequencies were computed. Moreover, individual beta and gamma frequencies were computed. Three frequency peaks were detected in the frequency range from the individual alpha 3 frequency band and $45 \mathrm{~Hz}$. These peaks were named betal peak (IBF 1), beta2 peak (IBF 2), and gamma peak (IGF). Based on peaks, the frequency ranges were determined. Betal ranges from alpha 3 to the lower spectral power value between betal and beta 2 peak; beta 2 frequency ranges from beta 1 to the lower spectral power value between beta2 and gamma peak; gamma frequency ranges from beta 2 to $45 \mathrm{~Hz}$, which is the end of the range considered. Moreover, within theta frequency the frequency peak (individual theta frequency, ITF) was also individuated. The mean frequency ranges computed in $\mathrm{AD} \mathrm{MCI}$ subjects considered as a whole are delta $2.7-4.7 \mathrm{~Hz}$, theta $4.7-6.7 \mathrm{~Hz}$, alpha1 $6.7-8.7 \mathrm{~Hz}$, alpha2 8.7-10.7 Hz, alpha3 $10.7-12-7 \mathrm{~Hz}$, beta1 $12.7-17.2 \mathrm{~Hz}$, beta2 17.2-30.4, gamma 30.4-45. In the frequency bands determined in this way, the relative power spectra for each subject was computed. The relative power density for each frequency band was computed as the ratio between the absolute power and the mean power spectra from 2 to $45 \mathrm{~Hz}$. The relative band power at each band was defined as the mean of the relative band power for each frequency bin within that band. For each subject all electrodes were pooled to obtain an all-scalp region relative power spectrum. Finally, alpha3/alpha2 relative power ratio were computed and analyzed.

2.2. Magnetic Resonance Acquisition. High-resolution gradient echo sagittal 3D MR scans were acquired with a Philips Gyroscan $1.0 \mathrm{~T}$ scanner $(\mathrm{TR}=20 \mathrm{~ms}$, $\mathrm{TE}=5 \mathrm{~ms}$, flip angle $30^{\circ}$, field of view $=220 \mathrm{~mm}$, acquisition matrix $=256 \times 256$, slice thickness $=1.3 \mathrm{~mm}$, no interslice gap) with a standard head coil. The MRI scanning of AD patients was compared with that of twenty-two healthy normal elderly, chosen as normal controls, in order to verify if correlation between EEG rhythms and hippocampal regions was present in atrophic or nonatrophic areas. 
2.3. Image Processing. The $3 \mathrm{D}$ images were reoriented along the AC-PC line and voxels below the cerebellum were removed with the MRIcro software (http://www.cabiatl.com/ $\mathrm{mricro} / \mathrm{mricro} / \mathrm{mricro}$.html).

In order to improve extraction of the cerebral cortex in areas adjacent to the cerebellum. The anterior commissure was manually set as the origin of the spatial coordinates for an anatomical normalization algorithm implemented in the Statistical Parametric Mapping (SPM99) software package (http://www.fil.ion.ucl.ac.uk/spm/). A 12-parameter affine transformation was used to normalize each image to a customized template in stereotaxic space, created from the MRI scans of 40 control subjects taken from a well-characterized MRI archive [21, 22]. Warping of one hippocampus to another was based on matching homologous points on a rectilinear surface mesh adapted to the structure boundary. The hippocampi were manually traced on the reoriented and normalized images. A single tracer (A.P.) outlined the hippocampal boundaries on contiguous coronal $1.0 \mathrm{~mm}$ thick sections following a standardized and validated protocol [23] using an interactive software program developed at the LONI (Laboratory of NeuroImaging), University of California at Los Angeles (http://www.loni.ucla.edu/Software/index.php). Tracings included the hippocampus proper, dentate gyrus, subiculum (subiculum proper and presubiculum), alveus, and fimbria. Each hippocampus comprised approximately 30-40 consecutive slices, and tracing took about 30 minutes per subject. Test-retest reliability on 20 subjects was good: intraclass correlation coefficients were 0.89 for the left and 0.87 for the right hippocampus.

2.4. Radial Atrophy Mapping. The 3D parametric surface mesh models were created from the manual tracings of hippocampal boundaries [24]. This procedure allows measurements to be made at corresponding surface locations in each subject, which are then compared statistically in $3 \mathrm{D}$ [25]. To assess hippocampal morphology, a medial curve was automatically defined as the $3 \mathrm{D}$ curve traced out by the centroid of the hippocampal boundary in each image slice. The radial size of each hippocampus at each boundary point was assessed by automatically measuring the radial $3 \mathrm{D}$ distance from the surface points to the medial curve defined for individual's hippocampal surface model. Distance field indexing local expansions or contractions in hippocampal surface morphology were statistically compared between groups at equivalent hippocampal surface points in $3 \mathrm{D}$ space [26].

2.5. Statistical Analysis. Correlation maps between EEG rhythms and hippocampal surface were computed. The correlation analysis between EEG rhythms and hippocampal volume was performed only in $13 \mathrm{AD}$ subjects; we otherwise compared hippocampal gray matter distribution maps between normal controls and AD patients in order to verify that the $\mathrm{AD}$ found correlations between EEG rhythms and hippocampal regions were present in areas where ADS are more atrophic than normal subjects.
TABLE 1: ANOVA results of demographic variables, that is, age, education, MMSE score, and neurophysiological EEG markers, that is alpha3/alpha2 ratio (see text for details).

\begin{tabular}{lccc}
\hline & Normal old & $\mathrm{AD}$ & $\begin{array}{c}P \text {-value } \\
\text { (ANOVA) }\end{array}$ \\
\hline $\begin{array}{l}\text { Number of subjects } \\
(\mathrm{f} / \mathrm{m})\end{array}$ & $22(14 / 6)$ & $13(8 / 5)$ & \\
Age (years) & $73.5 \pm 3.4$ & $76.2 \pm 2.3$ & .1 \\
Education (years) & $8.8 \pm 1.2$ & $4.6 \pm 0.9$ & .4 \\
MMSE & $29.2 \pm 1.4$ & $21.3 \pm 2.5$ & .01 \\
alpha3/alpha2 ratio & $0.9 \pm 0.08$ & $1.5 \pm 0.2$ & .02 \\
\hline
\end{tabular}

The correlation maps were generated on 3D models of the hippocampal formation where the dorsal and ventral surfaces can be appreciated. Zones with significant correlations were mapped onto the models based on an atlas where these are shown together with the corresponding MR sections [27, 28] (Figure 1). The correlations and the associated $P$-value maps were plotted onto a colour-coded model of the hippocampal surface. The statistical test for the correlations was computed using linear regression at each surface vertex on the hippocampus [29]. A surface point significance threshold of $P<.05$ was used to visualize the regional specificity of gray matter changes in the cortex. Set level correction for multiple comparisons was carried out by permutation testing at threshold of $P=.05$. Permutation tests are based on measuring the total area of the hippocampus with suprathreshold statistics, after setting the threshold at $P<.05$. To correct for multiple comparisons and assign an overall $P$-value to each $P$-map $[30,31]$ permutation tests were used to determine how likely the observed level of significant atrophy (proportion of suprathreshold statistics, with the threshold set at $P<.05$ ) within each $P$-map would occur by chance [29]. The number of permutations $N$ was chosen to be 100,000, to control the standard error SEp of omnibus probability $p$, which follows a binomial distribution $B(N, p)$ with known standard error [32]. When $N=8,000$, the approximate margin of error (95\% confidence interval) for $\mathrm{P}$ is around $5 \%$ of $\mathrm{P}[33$ ].

Sociodemographic differences between groups were estimated with analysis of variance (ANOVA) test. Significance was set to $P<.05$.

\section{Results}

Table 1 shows ANOVA results of demographic variables, that is, age, education, MMSE score, and neurophysiological EEG markers, that is alpha3/alpha2 ratio. Significant results were obtained only for MMSE $(P<.01)$ with significant decrease in $\mathrm{AD}$ as compared to normal old subjects, and for alpha3/alpa2 ratio $(P<.02)$ with significant increase in $\mathrm{AD}$ as compared to normal old subjects.

Figure 2 displays the pattern of negative significant associations between alpha 3 rhythm and gray matter volume in the hippocampus; areas of strongest and significant $(r>$ $-.40, P<.05)$ correlation mapped to little spots in the left 


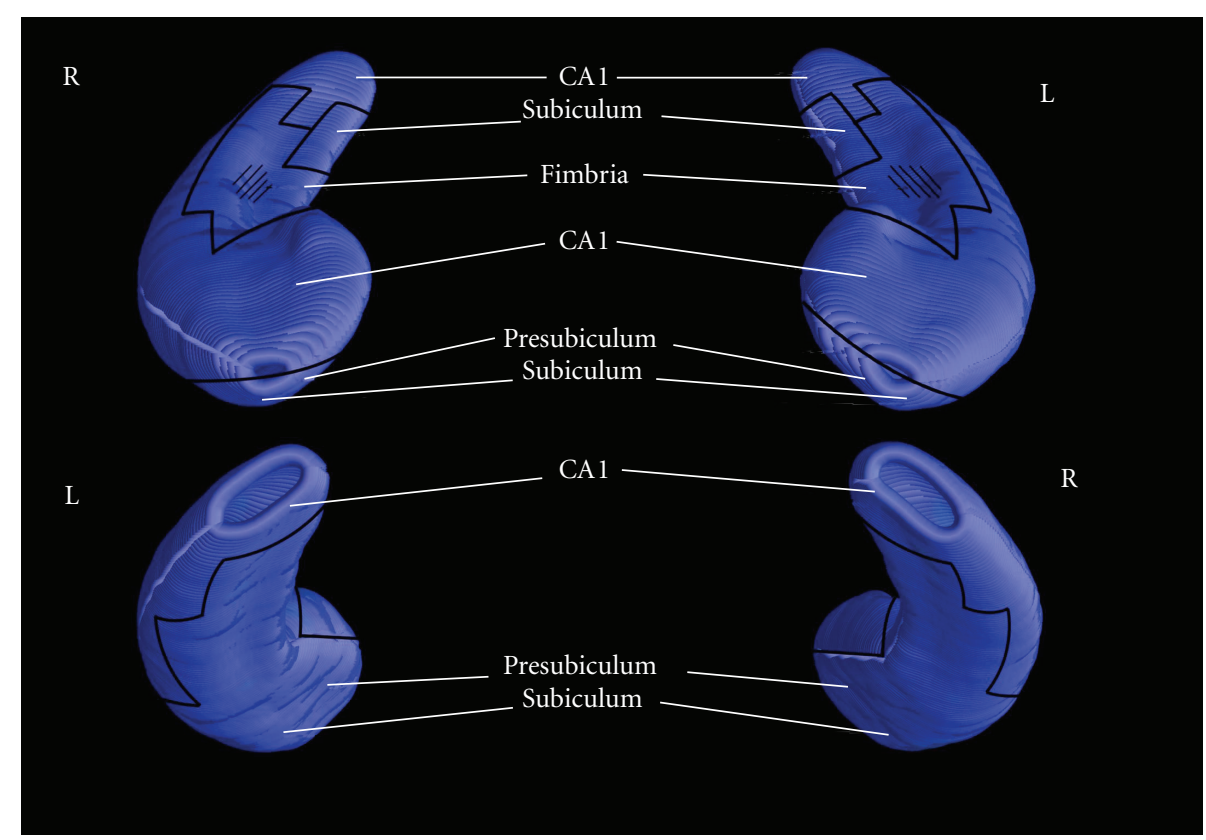

FIGURE 1: Cytoarchitectonic subregions mapped on blank MR-based models of the hippocampal formation of a healthy subject.

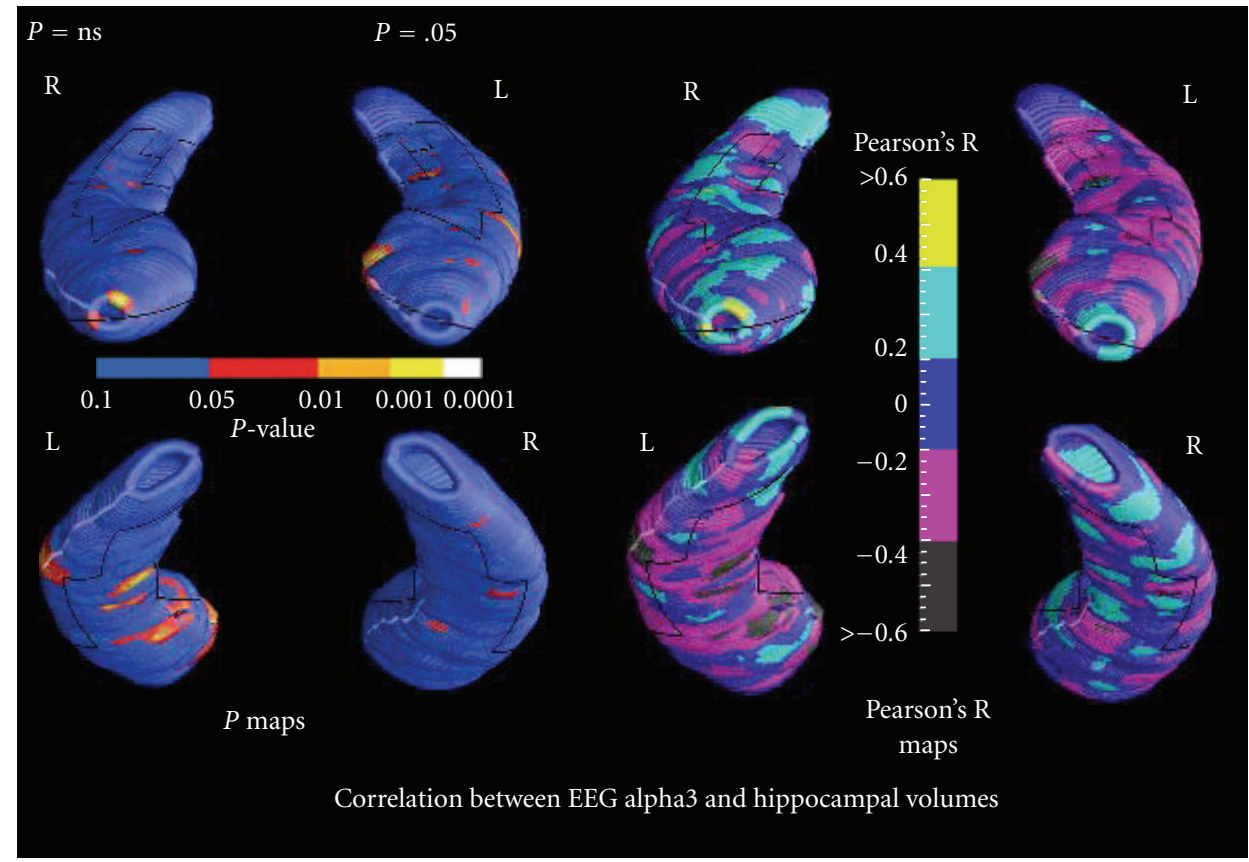

FIGURE 2: Correlation between alpha3 power rhythm and volumes of hippocampal subregions in AD patients. Permutations overall $P$ values are displayed on top of each hippocampus.

dorsal subiculum and CA2-3 sectors of the body and to the CA1 mesial and lateral portion of the head; moreover, large areas of significant correlations were distributed across widespread left ventral subiculum and presubiculum.

Figure 3 shows correlations between alpha3/alpha2 rhythms ratio and hippocampal volumes in $\mathrm{AD}$ patients. Again, negative significant associations are found between same areas of AD left hippocampus, although a bit enlarged, and alpha3/2 EEG rhythms. Correlations in right hippocampus resulted not significant at permutation testing $(P>.75$ in both cases).

Although small areas of significant correlation between EEG rhythms and volumes were detected in right hippocampus too, the overall $P$-maps resulted globally not significant ( $P$ at permutation testing $>.05$ as stated at top of Figures 2 and 3); so we cannot exclude the null hypothesis that for right 


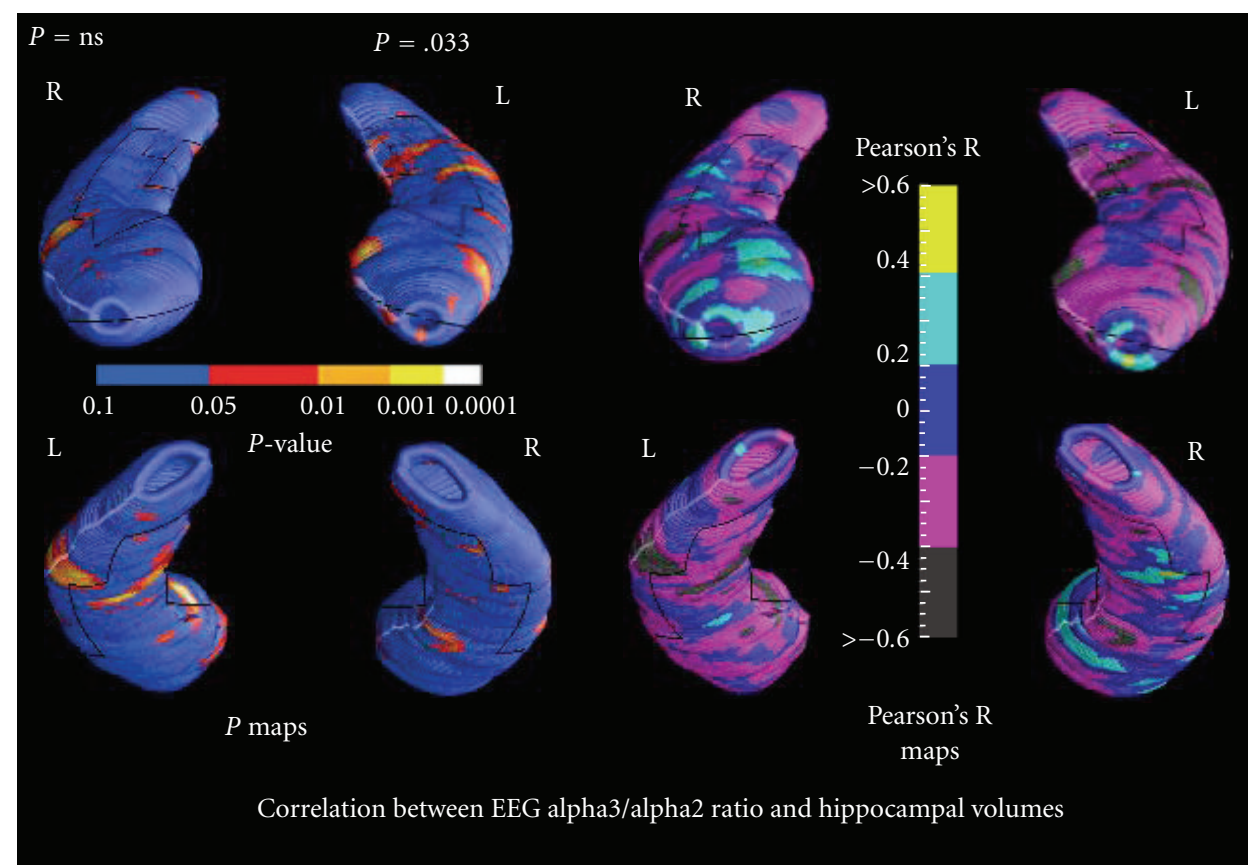

FIGURE 3: Correlation between alpha3/alpha2 power rhythm ratio and volumes of hippocampal subregions in $\mathrm{AD}$ patients. Permutations overall $P$ values are displayed on top of each hippocampus.

hippocampus only, all correlations detected occurred by chance. Moreover, for both hippocampi, areas of significant positive correlations are really small and, moreover, localized in a zone, at the very start of the head of the structure, that is usually not taken into account in radial atrophy map analyses. This area could be more often affected by manual tracing bias and systematic bias due to changes in the anatomy of the hippocampus of patients and difficult in well discriminating boundaries between low contrast structures. Some of the bias derive from the resolution of the scanner, in that the voxel size is of the order of $1 \mathrm{~mm}$. Other sources of error derive from the manual tracing method although the reliability for the method has been shown to be high [26].

The hippocampal areas of correlation are all atrophic in $\mathrm{AD}$ patients as compared to normal controls.

\section{Discussion}

The main result of the present study is the finding of a correlation between brain electrical activity collected by scalp EEG and discrete-mapped hippocampal areas in subjects with AD.

The results show that in $\mathrm{AD}$ patients the increase of both alpha 3 rhythm spectral power and alpha3/alpha2 power ratio is correlated with the decrease of left hippocampal gray matter volumes.

Our findings confirm the outcomes of two recent metaanalyses. Indeed, a metaanalysis of voxel-based morphometry studies [34], analyzing 429 subjects with amnesic mild cognitive impairment (aMCI), found that the left medial temporal lobe atrophy is the most consistent neurostruc- tural biomarker to predict conversion from aMCI to AD. Moreover a recent metaanalysis focused on the left-right hippocampal pattern asymmetry in $\mathrm{AD}$ [35] has considered 28 studies, in which 23 studies included $700 \mathrm{AD}$ patients and 751 controls, 14 studies included 365 MCI patients and 382 controls, and nine studies contain both AD and MCI. The results have demonstrated that the asymmetry in the left and right hippocampal volume, with a less-than-right pattern, exists and may vary with disease progression.

In particular, our results showed that hippocampal areas involved in correlation with EEG markers are presubiculum, dorsal and ventral subiculum, CA2-3 sectors of the body, and CA1 mesial and lateral portion of the head. These findings confirm previous results obtained in a large cohort of patient with mild cognitive impairment (MCI) who convert in AD. Indeed, the increase of alpha 3 band power was found in MCI patient with hippocampal atrophy [7]. Moreover, the increase of alpha3/alpha2 power ratio is the most specific EEG marker of hippocampal atrophy $[10,11]$, and it is highly predictive of MCI who will convert to AD (12 Moretti et al. in press). Moreover, the prevalence of the modification of EEG rhythms in the left hemisphere in patients with incipient AD was found also in a recent EEG coherence study [8].

The hippocampus is affected early in Alzheimer's disease by neurofibrillary tangle deposition, spreading from the entorhinal cortex to the CA1 subfield, and subicular region, then to the CA2-3 subfields, the CA4 subfield and finally the neocortex [36].

The atrophy of the hippocampal subregions in $\mathrm{AD}$ patients seems to affect the polysynaptic pathway of the traditional hippocampal circuitry. This pathway is characterized 
by projections by dentate gyrus to CA $2 / 3$ region, through the mossy fiber pathway. The CA3 Schaffer collaterals project to CA1 and, lastly, CA1 projects to the subiculum. This internal circuit of hippocampus is involved in processing details of information through pattern separation and pattern completion processes. In turn, autoassociative networks of the hippocampal subregions, such as that present in CA3 (but also in CA1), have been proposed to be essential for encoding, filtering, and storing memories [37, 38]. This filter or storage function information relies on the integrity of synchronization of the temporal dynamics.

The synchronization of high alpha power has been demonstrated to be involved in top-down cognitive processes [39]. This finding could suggest that there is an the attempt to focus attention on highly selective aspect to prevent interference of irrelevant stimuli (top-down process) in order to maintain a good memory performance $[7,39]$. Recently [7], we suggest that MCI subjects could fall in a "hyperattentive state" during the course of disease. Our results confirm this hypothesis, extending those findings to $\mathrm{AD}$ patients.

The correlation of the increase of alpha 3 power and alpha3/alpha2 power ratio with atrophic areas could suggest that there is a compensatory synchronization in high alpha rhythm in an effort to balance the degenerative process. Another possible explanation is that the disruption of the order of a stable attractor network prevents the synchronization of large neural assemblies, mirrored by the low alpha synchronization, inducing an increase of high alpha power.

The specific involvement of alpha rhythm and the absence of significant findings in other rhythms, such as theta frequency, could suggest that the hippocampal atrophy in $\mathrm{AD}$ is linked to functional changes in a broader network. Indeed, alpha rhythm is not specific of hippocampus like theta frequency. Generation of alpha rhythm arises from thalamus-posterior cortical areas interplay. Of note, the hypometabolism and atrophy of posterior cingulate/retrosplenial and medial temporal cortex pathway, strictly connected with both hippocampus and visual cortex, as well as with low alpha rhythm generation, is well demonstrated in $\mathrm{AD}[40]$. So, the prevalence of high alpha could underlie the disruption of extensive synaptic connection deriving in the formation of smaller cell assemblies. The impairment of the network could explain memory and cognitive symptoms of $\mathrm{AD}$ beyond the hippocampal atrophy itself.

\section{Conclusions}

Our findings confirm the possible diagnostic role of EEG markers when integrated with morphostructural measures in patients with $\mathrm{AD}$.

\section{Conflict of Interests}

Authors have no conflict of interests.

\section{References}

[1] G. B. Frisoni, R. Ganzola, E. Canu et al., "Mapping local hippocampal changes in Alzheimer's disease and normal ageing with MRI at 3 Tesla," Brain, vol. 131, no. 12, pp. 32663276, 2008.

[2] A. C. Papanicolaou, P. G. Simos, E. M. Castillo, J. I. Breier, J. S. Katz, and A. A. Wright, "The hippocampus and memory of verbal and pictorial material," Learning and Memory, vol. 9, no. 3, pp. 99-104, 2002.

[3] B. A. Strange, P. C. Fletcher, R. N. A. Henson, K. J. Friston, and R. J. Dolan, "Segregating the functions of human hippocampus," Proceedings of the National Academy of Sciences of the United States of America, vol. 96, no. 7, pp. 4034-4039, 1999.

[4] C. J. Stam, Y. van der Made, Y. A. L. Pijnenburg, and P. H. Scheltens, "EEG synchronization in mild cognitive impairment and Alzheimer's disease," Acta Neurologica Scandinavica, vol. 108, no. 2, pp. 90-96, 2003.

[5] C. Babiloni, G. Binetti, E. Cassetta et al., "Sources of cortical rhythms change as a function of cognitive impairment in pathological aging: a multicenter study," Clinical Neurophysiology, vol. 117, no. 2, pp. 252-268, 2006.

[6] D. V. Moretti, C. Miniussi, G. Frisoni et al., "Vascular damage and EEG markers in subjects with mild cognitive impairment," Clinical Neurophysiology, vol. 118, no. 8, pp. 1866-1876, 2007.

[7] D. V. Moretti, C. Miniussi, G. B. Frisoni et al., "Hippocampal atrophy and EEG markers in subjects with mild cognitive impairment," Clinical Neurophysiology, vol. 118, no. 12, pp. 2716-2729, 2007.

[8] D. V. Moretti, G. B. Frisoni, M. Pievani et al., "Cerebrovascular disease and hippocampal atrophy are differently linked to functional coupling of brain areas: an EEG coherence study in MCI subjects," Journal of Alzheimer's Disease, vol. 14, no. 3, pp. 285-299, 2008.

[9] D. V. Moretti, M. Pievani, C. Fracassi et al., "Brain vascular damage of cholinergic pathways and EEG markers in mild cognitive impairment," Journal of Alzheimer's Disease, vol. 15, no. 3, pp. 357-372, 2008.

[10] D. V. Moretti, C. Fracassi, M. Pievani et al., "Increase of theta/gamma ratio is associated with memory impairment," Clinical Neurophysiology, vol. 120, no. 2, pp. 295-303, 2009.

[11] D. V. Moretti, M. Pievani, C. Fracassi et al., "Increase of theta/Gamma and Alpha3/Alpha2 ratio is associated with amygdalo-hippocampal complex atrophy," Journal of Alzheimer's Disease, vol. 17, no. 2, pp. 349-357, 2009.

[12] D. V. Moretti, G. B. Frisoni, C. Fracassi et al., "MCI patients' EEGs show group differences between those who progress and those who do not progress to AD," Neurobiology of Aging, vol. 32, no. 4, pp. 563-571, 2011.

[13] G. McKhann, D. Drachman, and M. Folstein, "Clinical diagnosis of Alzheimer's disease: report of the NINCDS-ADRDA work group under the auspices of Department of Health and Human Services Task Force on Alzheimer's disease," Neurology, vol. 34, no. 7, pp. 939-944, 1984.

[14] APA. American Psychiatric Association, Diagnostic and Statistical Manual of Mental Disorders, American Psychiatric Publishing, Arlington, Va, USA, 4th edition, 1994.

[15] D. V. Moretti, F. Babiloni, F. Carducci et al., "Computerized processing of EEG-EOG-EMG artifacts for multi-centric studies in EEG oscillations and event-related potentials," International Journal of Psychophysiology, vol. 47, no. 3, pp. 199-216, 2003. 
[16] F. H. Lopes da Silva, J. P. Pijn, D. Velis, and P. C. G. Nijssen, "Alpha rhythms: noise, dynamics and models," International Journal of Psychophysiology, vol. 26, no. 1-3, pp. 237-249, 1997.

[17] P. L. Nunez, B. M. Wingeier, and R. B. Silberstein, "Spatial-temporal structures of human alpha rhythms: theory, microcurrent sources, multiscale measurements, and global binding of local networks," Human Brain Mapping, vol. 13, no. 3, pp. 125-164, 2001.

[18] S. Yuval-Greenberg, O. Tomer, A. S. Keren, I. Nelken, and L. Y. Deouell, "Transient induced gamma-band response in EEG as a manifestation of miniature saccades," Neuron, vol. 58, no. 3, pp. 429-441, 2008.

[19] W. Klimesch, "EEG alpha and theta oscillations reflect cognitive and memory performance: a review and analysis," Brain Research Reviews, vol. 29, no. 2-3, pp. 169-195, 1999.

[20] D. V. Moretti, C. Babiloni, G. Binetti et al., "Individual analysis of EEG frequency and band power in mild Alzheimer's disease," Clinical Neurophysiology, vol. 115, no. 2, pp. 299-308, 2004.

[21] R. Riello, F. Sabattoli, A. Beltramello et al., "Brain volumes in healthy adults aged 40 years and over: a voxel-based morphometry study," Aging Clinical and Experimental Research, vol. 17, no. 4, pp. 329-336, 2005.

[22] G. B. Frisoni, M. Pievani, C. Testa et al., "The topography of grey matter involvement in early and late onset Alzheimer's disease," Brain, vol. 130, no. 3, pp. 720-730, 2007.

[23] J. C. Pruessner, L. M. Li, W. Serles et al., "Volumetry of hippocampus and amygdala with high-resolution MRI and threedimensional analysis software: minimizing the discrepancies between laboratories," Cerebral Cortex, vol. 10, no. 4, pp. 433 $442,2000$.

[24] K. L. Narr, P. M. Thompson, P. Szeszko et al., "Regional specificity of hippocampal volume reductions in first-episode schizophrenia," NeuroImage, vol. 21, no. 4, pp. 1563-1575, 2004.

[25] P. M. Thompson, C. Schwartz, and A. W. Toga, "Highresolution random mesh algorithms for creating a probabilistic 3D surface atlas of the human brain," NeuroImage, vol. 3, no. 1, pp. 19-34, 1996.

[26] P. M. Thompson, K. M. Hayashi, G. I. De Zubicaray et al., "Mapping hippocampal and ventricular change in Alzheimer disease," NeuroImage, vol. 22, no. 4, pp. 1754-1766, 2004.

[27] H. M. Duvernoy, Ed., The Human Hippocampus. Functional Anatomy, Vascularization and Serial Section with MRI, Springer, Berlin, Germany, 3rd edition, 1998.

[28] G. B. Frisoni, F. Sabattoli, A. D. Lee, R. A. Dutton, A. W. Toga, and P. M. Thompson, "In vivo neuropathology of the hippocampal formation in AD: a radial mapping MR-based study," NeuroImage, vol. 32, no. 1, pp. 104-110, 2006.

[29] P. M. Thompson, K. M. Hayashi, G. de Zubicaray et al., "Dynamics of gray matter loss in Alzheimer's disease," Journal of Neuroscience, vol. 23, no. 3, pp. 994-1005, 2003.

[30] T. E. Nichols and A. P. Holmes, "Nonparametric permutation tests for functional neuroimaging: a primer with examples," Human Brain Mapping, vol. 15, no. 1, pp. 1-25, 2002.

[31] E. S. Edgington, Randomization Tests, Marcel Dekker, New York, NY, USA, 1995.

[32] E. S. Edgington and P. Onghena, Randomization Tests, Chapman \& Hall/CRC, Boca Raton, Fla, USA, 2007.

[33] J. H. Morra, Z. Tu, L. G. Apostolova et al., "Automated 3D mapping of hippocampal atrophy and its clinical correlates in 400 subjects with Alzheimer's disease, mild cognitive impairment, and elderly controls," Human Brain Mapping, vol. 30, no. 9, pp. 2766-2788, 2009.

[34] L. K. Ferreira, B. S. Diniz, O. V. Forlenza, G. F. Busatto, and M. V. Zanetti, "Neurostructural predictors of Alzheimer's disease: a meta-analysis of VBM studies," Neurobiology of Aging. In press.

[35] F. Shi, B. Liu, Y. Zhou, C. Yu, and T. Jiang, "Hippocampal volume and asymmetry in mild cognitive impairment and Alzheimer's disease: meta-analyses of MRI studies," Hippocampus, vol. 19, no. 11, pp. 1055-1064, 2009.

[36] B. Schönheit, R. Zarski, and T. G. Ohm, "Spatial and temporal relationships between plaques and tangles in Alzheimerpathology," Neurobiology of Aging, vol. 25, no. 6, pp. 697-711, 2004.

[37] O. C. Zalay and B. L. Bardakjian, "Simulated mossy fiber associated feedforward circuit functioning as a highpass filter," in Proceedings of the 28th Annual International Conference of the IEEE Engineering in Medicine and Biology Society (EMBS '06), pp. 4979-4982, September 2006.

[38] N. M. van Strien, N. L. M. Cappaert, and M. P. Witter, "The anatomy of memory: an interactive overview of the parahippocampal- hippocampal network," Nature Reviews Neuroscience, vol. 10, no. 4, pp. 272-282, 2009.

[39] W. Klimesch, P. Sauseng, and S. Hanslmayr, "EEG alpha oscillations: the inhibition-timing hypothesis," Brain Research Reviews, vol. 53, no. 1, pp. 63-88, 2007.

[40] G. B. Frisoni, A. Prestia, P. E. Rasser, M. Bonetti, and P. M. Thompson, "In vivo mapping of incremental cortical atrophy from incipient to overt Alzheimer's disease," Journal of Neurology, vol. 256, no. 6, pp. 916-924, 2009. 


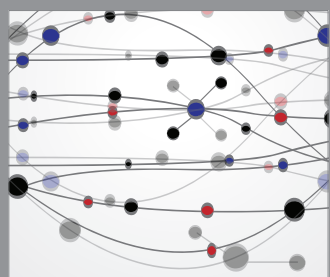

The Scientific World Journal
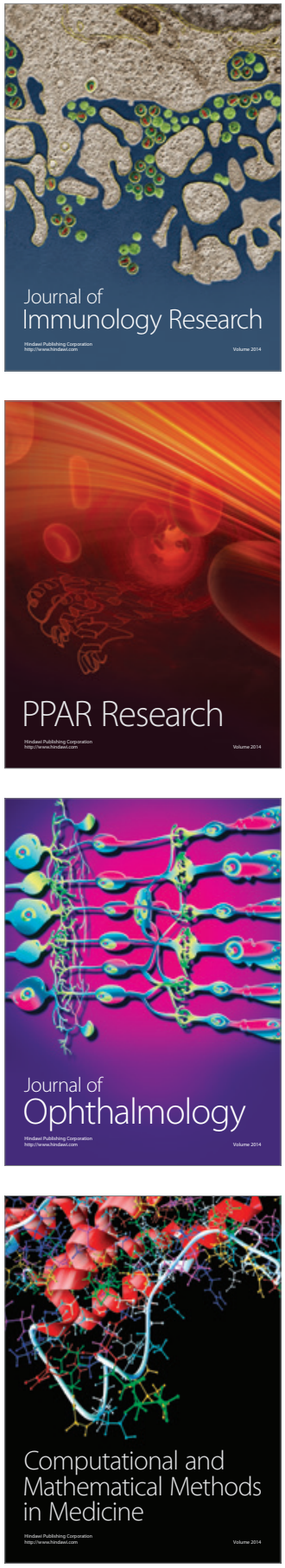

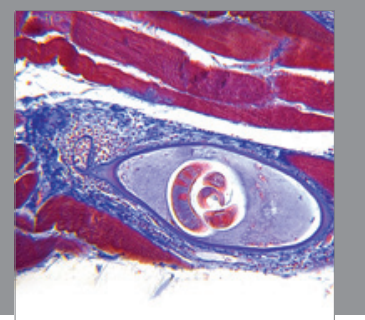

Gastroenterology

Research and Practice
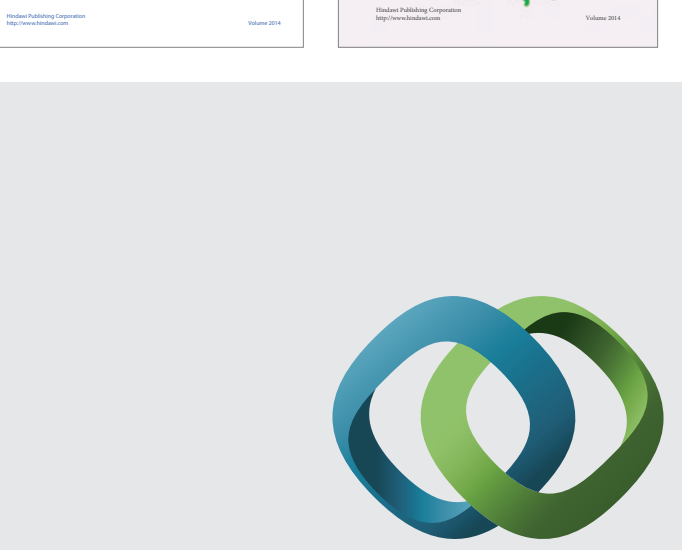

\section{Hindawi}

Submit your manuscripts at

http://www.hindawi.com
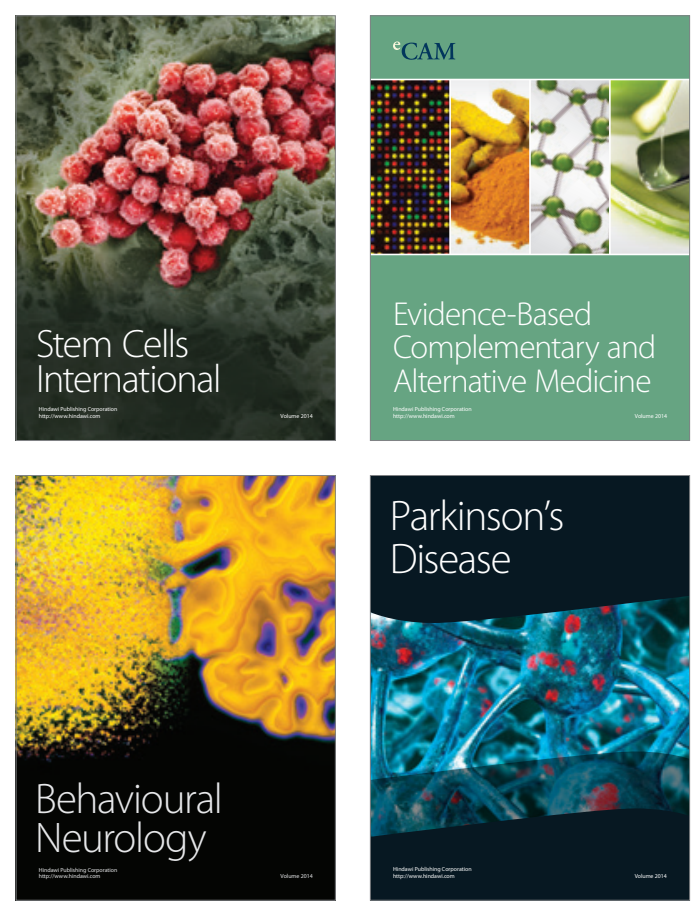

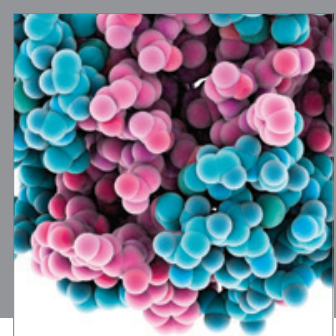

Journal of
Diabetes Research

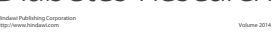

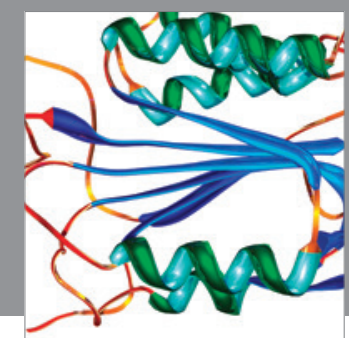

Disease Markers
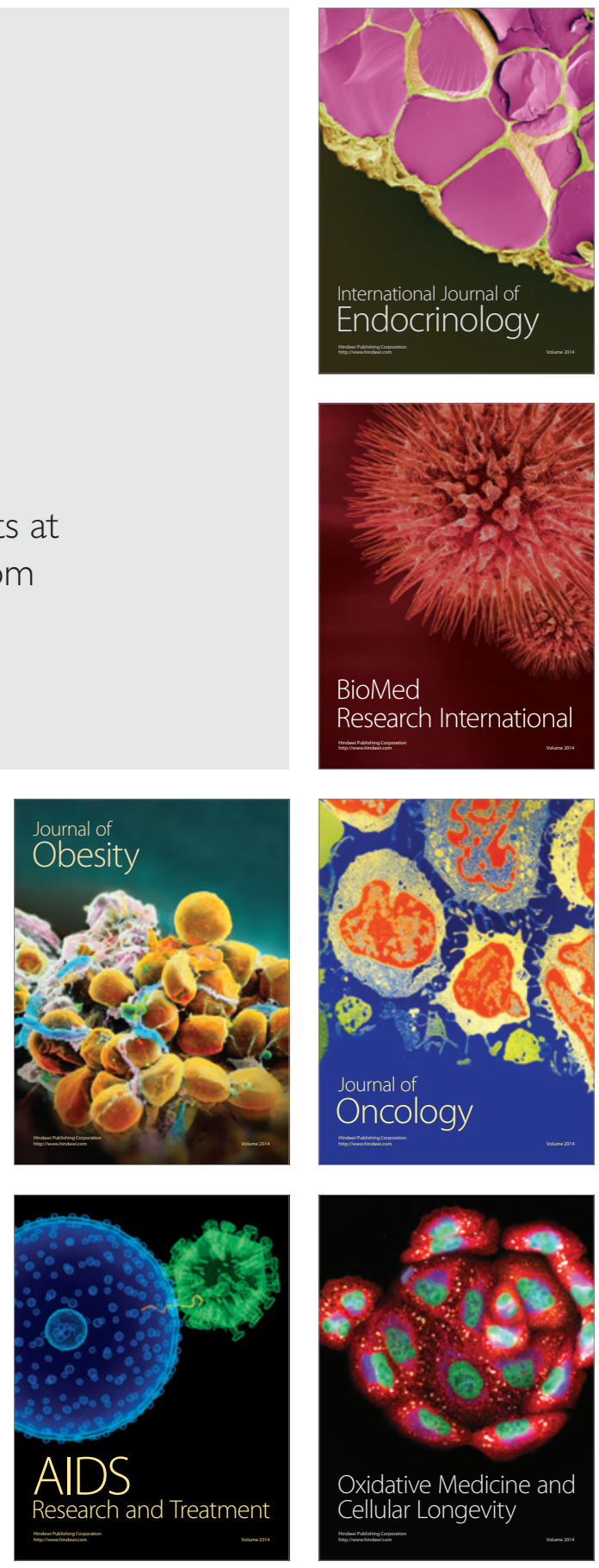